\title{
Correction to: Machine Learning to Analyze Single-Case Data: A Proof of Concept
}

\author{
Marc J. Lanovaz ${ }^{1} \cdot$ Antonia R. Giannakakos $^{2} \cdot$ Océane Destras $^{3}$ \\ Published online: 10 February 2021 \\ (C) Association for Behavior Analysis International 2021
}

\section{Correction to: Perspectives on Behavior Science https://doi.org/10.1007/s40614-020-00244-0}

The equation on page 33 should be replaced by the following:

"We used R code to generate 20,000 AB graphs with three points in Phase A and five points in Phase B. The $\mathrm{R}$ code generated an eight-point data series for each graph using the equations:

$$
y_{t}=x_{t}+c
$$

$$
\text { where } x_{t}=a x_{t-1}+\varepsilon_{t}
$$

In the previous equations, $y$ represented a univariate time series, $t$ an index of time (i.e., one to eight), $a$ the autocorrelation value, $\varepsilon$ a normally distributed error term with a mean of 0 and a standard deviation of 1 , and $c$ a constant of 10 to avoid graphs with negative values."

The online version of the original article can be found at https://doi.org/10.1007/s40614-020-00244-0

Marc J. Lanovaz

marc.lanovaz@umontreal.ca

1 École de Psychoéducation, Université de Montréal, C.P. 6128, succursale Centre-Ville, Montreal, QC H3C 3J7, Canada

2 Manhattanville College, Purchase, NY, USA

3 Polytechnique Montréal, Montréal, QC, Canada 